\title{
Digital Processing with a Hybrid Plasmonic Logic Nanogate
}

Laraa Al Nubani, Nicholas Dogantzis, Gregory Hodgson, Stefania Impellizzeri*

Ryerson University, Department of Chemistry \& Biology

\section{ABSTRACT}

We developed a simple strategy to gate a plasmonic event with a hybrid photoresponsive compound capable of mimicking AND-type logic behaviour. Binary digits are encoded into ultraviolet and visible illumination (which are used as inputs) and the fluorescence of the molecule (the arithmetic output) The plasmonic process chosen is the visible lightcontrolled, gold-nanoparticle (AuNP) catalysed reduction of resazurin to resorufin, while the second optical input is the UVA-induced cleavage of a 2nitrobenzyl quencher. The synergistic combination of plasmonic nanostructures and organic molecules allows for the manipulation of a programmable Boolean logic operation at the molecular level.

AuNP SYNTHESIS

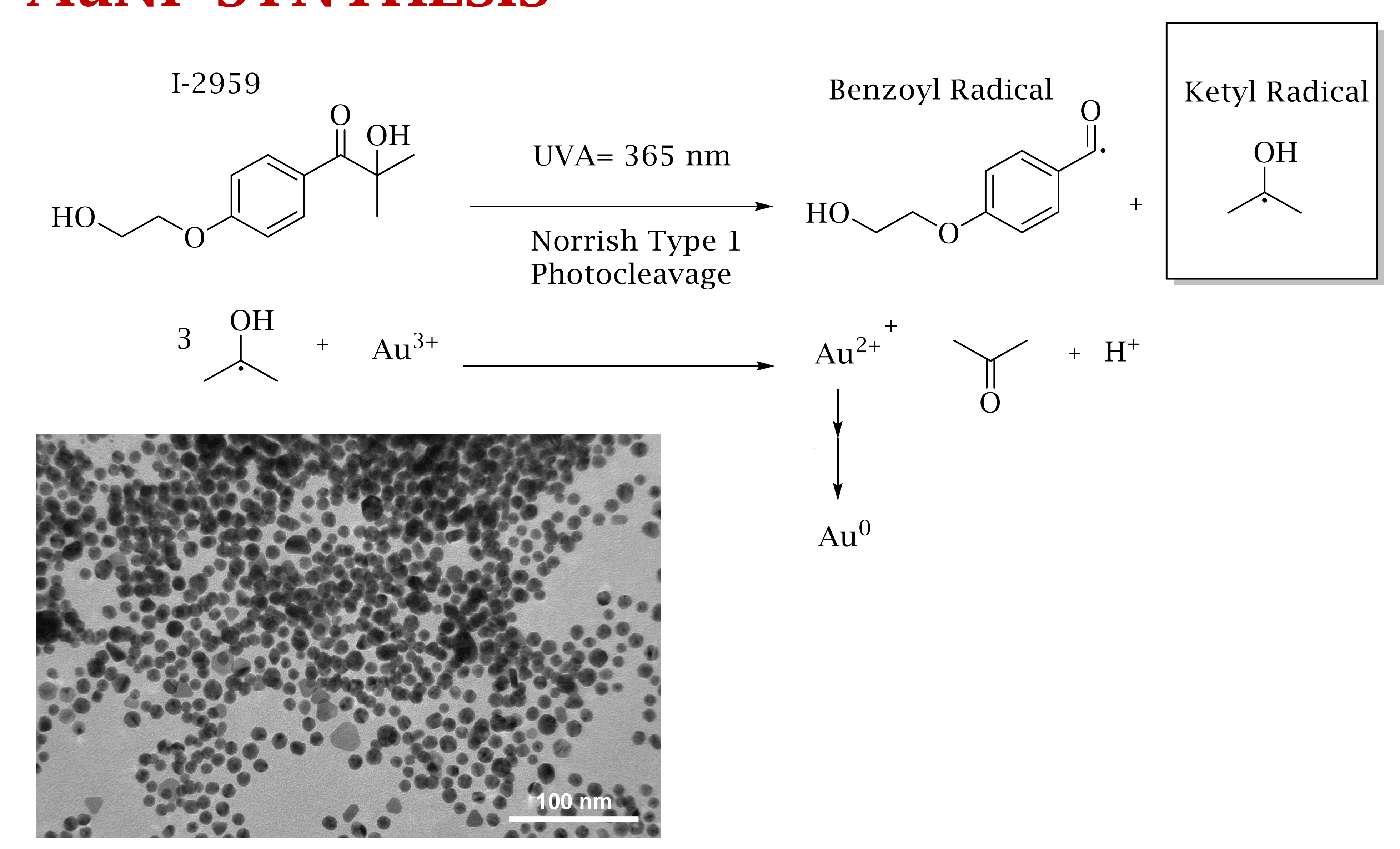

SYNTHESIS OF PHOTOCAGED COMPOUNDS

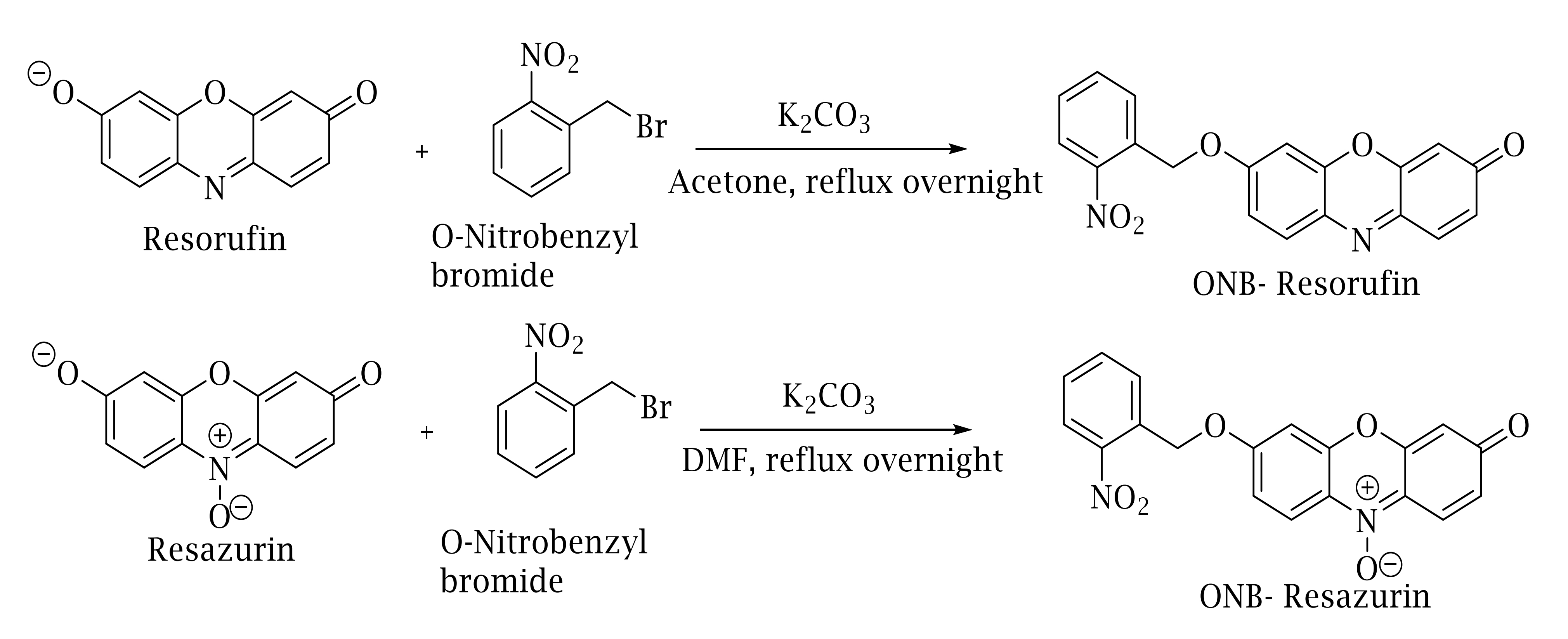

\section{PROJECT GOAL}

Transducing Logic Gate Behaviour to the Molecular Level using a Photoresponsive Compound and Photochemical Inputs
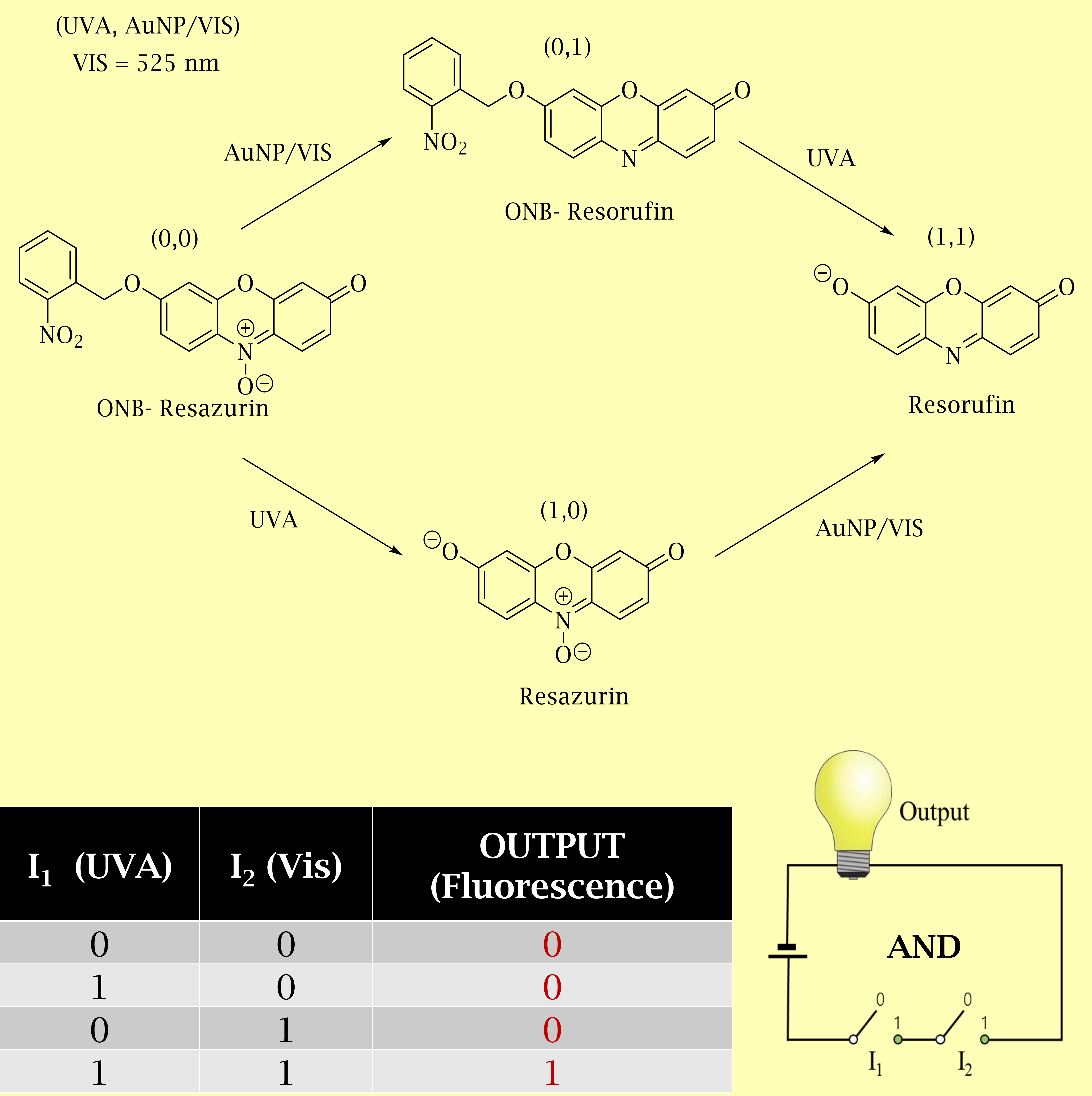

Table 1. Truth table and circuit of the AND logic gate.
RESULTS
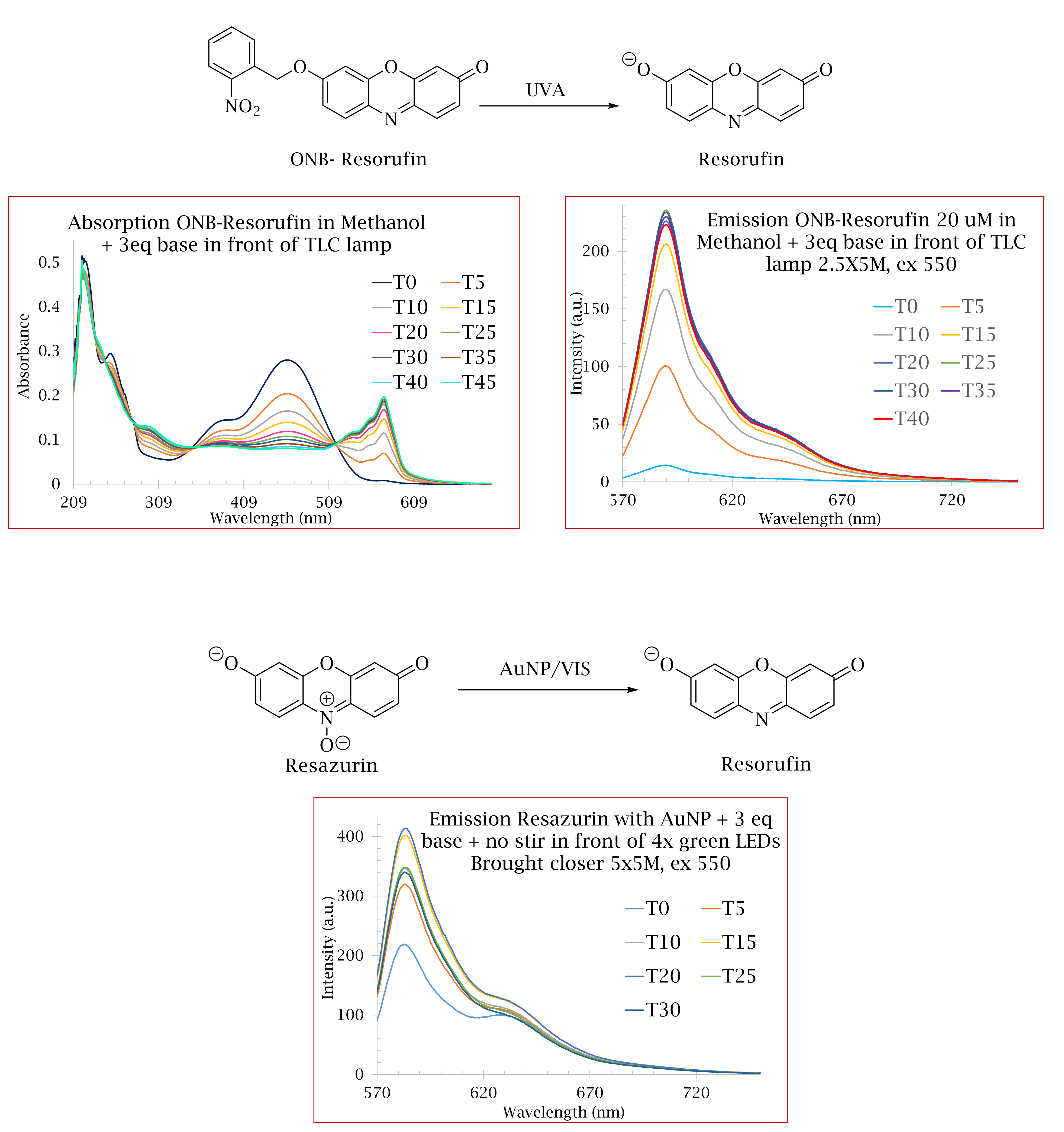

REFERENCES

Mcgilvray, K. L.; Decan, M. R.; Wang, D.; Scaiano, J. C. Journal of the American Chemical Society 2006, 128, 15980.

Alejo, C. J. B., Fascini, C. Grenier, Mi, Netto-Ferreira, J. C, Scairno, J. C. Catalysis Science \& Technology 2011, 1, 1506.

\section{ACKNOWLEDGMENTS}

The Undergraduate Research Opportunities (URO) Program

ana Al Nubani, Wesam A., Zainab Shakeel and Melissa D'Amaral

The members of Drs. Foucher, Gossage and McWilliams Lab

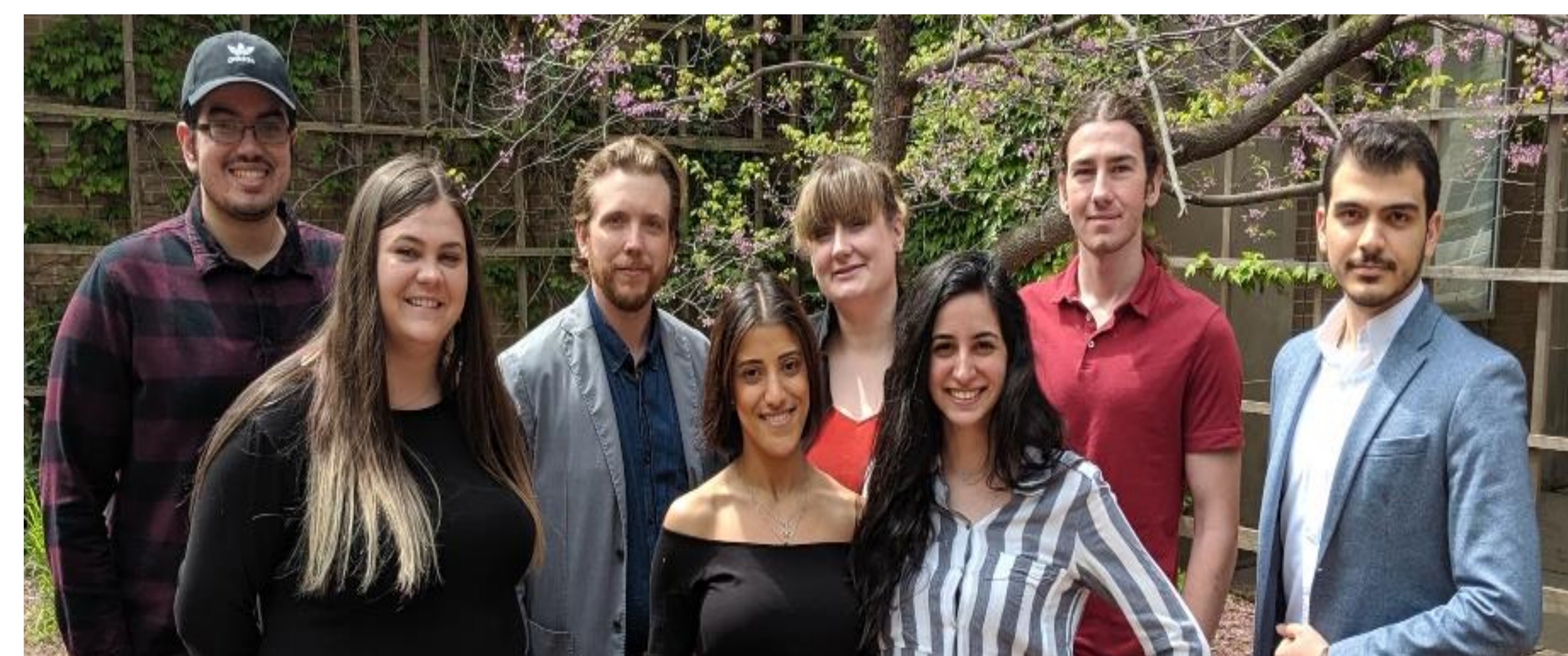

yerson 